\title{
THE EFFECTIVE TEMPERATURES OF HOT STARS
}

\author{
P.A. CROWTHER \\ Dept. of Physics \& Astronomy, University College London, \\ Gower Street, London, WC1E 6BT, U.K.
}

\begin{abstract}
We review the effective temperature scale of hot $(\geq 10 \mathrm{kK})$ stars, including results from direct, continuum and ionization equilibrium techniques. We discuss the impact of recent developments in theoretical model atmospheres for OB subdwarfs, dwarfs and supergiants, white dwarfs and Wolf Rayet stars and present a revised $T_{\text {eff }}$ scale for OB stars. Direct techniques coupled with Kurucz model atmospheres allow Strömgren photome-

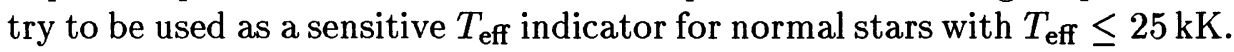
Reliable $T_{\text {eff }}$ determinations for hotter, low surface gravity and H-deficient stars require sophisticated ionization equilibrium techniques, generally considering non-LTE and line-blanketing effects.
\end{abstract}

Key words: stars:temperatures - early-type - fundamental parameters

\section{Continuum techniques}

\subsection{DIRECT METHODS}

The effective temperature of a star - providing the essential link between observed properties and results of theoretical stellar structure and atmosphere models - can be directly obtained from measurements of its angular diameter together with the total absolute flux at the Earth, integrated over the entire spectrum The prime source in this field remains the seminal work of Code et al. (1976), who combined angular diameters measured by the Narrabri stellar intensity interferometer with UV, visual and IR observations for 32 hot stars, corrected for interstellar extinction. Smalley \& Dworetsky (1995) repeated their analysis using more recent observational and theoretical results, and obtained values on average only one percent lower for stars with $T_{\text {eff }} \leq 25 \mathrm{kK}$.

T.R. Bedding et al. (eds.),

Fundamental Stellar Properties: The Interaction between Observation and Theory, 137-146.

(C) 1997 IAU. Printed in the Netherlands. 
While this method is termed 'direct', the allowance for fluxes in the unobservable Lyman continuum are taken from model calculations, and so becomes increasingly reliant on suitable models for very hot stars. For $\mathrm{O}$ and early B stars a significant fraction of the total energy is emitted shortward of $\lambda=912 \AA$ and obtaining $T_{\text {eff }}$ from observed energy distributions requires accurate knowledge of surface gravities (Abbott \& Hummer 1985; Hummer et al. 1988). Therefore, more sophisticated techniques need to be employed for such stars. We now turn to indirect techniques used for the temperature determination in the vast majority of hot stars.

\subsection{CONTINUUM FLUX METHODS}

The infrared flux method is a semi-direct technique developed by Blackwell \& Shallis (1977) for cool stars, which uses the ratio of the integrated flux relative to a monochromatic IR flux to obtain a star's angular diameter and $T_{\text {eff }}$ using an iterative procedure based on model atmospheres. Napiwotzki et al. (1993) found that the V-band flux provided an better calibration for B, $\mathrm{A}$ and $\mathrm{F}$ stars. Fitting model spectra to observations over the entire spectral range provides improved results for B stars (e.g. Malagnini et al. 1986; Drilling et al. 1984). Temperatures for DB white dwarfs can be obtained from UV continuum fits (e.g. Thejll et al. 1991), while hotter stars tend to be poorly constrained (e.g. ' $R$-index' technique of Schönberner \& Drilling 1984), often suffering the added complication of varying UV interstellar extinctions from star to star.

\subsection{PHOTOMETRIC METHODS}

Strömgren $u v b y \beta$ photometry, coupled with $\operatorname{Kurucz}(1979,1991)$ model atmospheres, provides a powerful technique for the determination of $T_{\text {eff }}$ in B, A and F stars. Napiwotzki et al. (1993) have provided a discussion of the various calibrations developed over the past decade, with the reddening free Strömgren colour-indices $\left[c_{1}\right]$ and $[u-b]$ widely employed. Fig. 1 demonstrates the temperature sensitivity of the Balmer jump for B stars based on Kurucz model atmospheres, and includes the Strömgren filter bandpasses. Temperatures of hot stars are poorly constrained using the Johnson reddening free index (e.g. Moehler et al. 1990).

Photometric calibrations applied to supergiants and stars showing nonsolar He-contents can lead to significant discrepancies since their colours typically lie either at the edge of, or beyond current calibrations and the strength of their Balmer jumps are affected by composition effects (Kudritzki et al. 1989). For example, Venn (1995) obtained $T_{\text {eff }}=9.7 \mathrm{kK}$ from a detailed spectral analysis of HD $46300(\mathrm{~A} 0 \mathrm{Ib})$ using $\mathrm{Mg}$ I-II lines, which compares with $8.4 \mathrm{kK}$ from the $(b-y)_{0}$ calibration of Napiwotzki et al. 


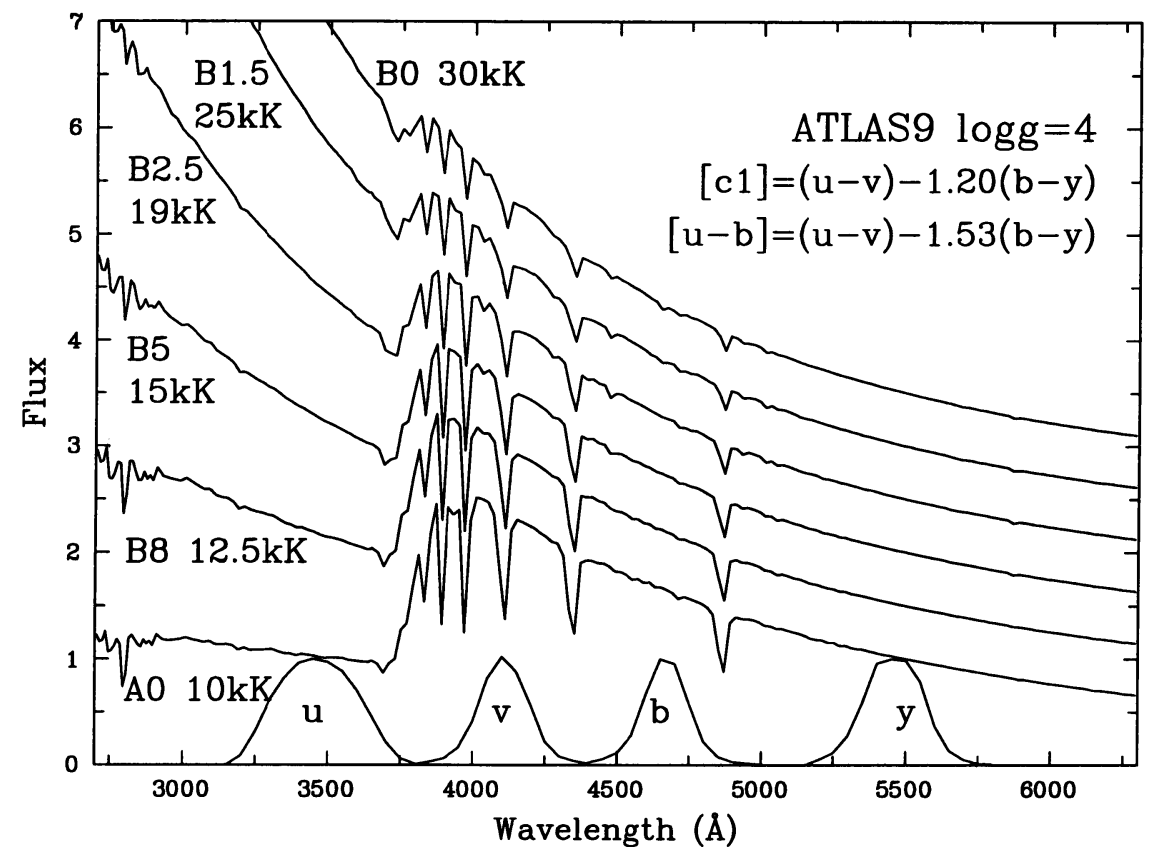

Figure 1. Kurucz (ATLAS9) theoretical energy distributions for B0-A0 dwarfs showing the strong temperature sensitivity of the Balmer jump, measured by the Strömgren colour-indices $\left[c_{1}\right]$ (favoured below $\sim 20 \mathrm{kK}$ ) and $[u-b]$ (above $\sim 20 \mathrm{kK}$ )

(1993), and $10.0 \mathrm{kK}$ from the $\beta$ versus [ $\left.c_{1}\right]$ relation of Lester et al. (1986). Saffer et al. (1994) have discussed the systematic errors obtained using standard photometric calibrations (e.g. Lester et al. 1986) for temperatures of (He-poor) sdB stars.

\section{Spectroscopic techniques}

While line blanketed, LTE Kurucz model atmospheres are widely employed for early-type stars, the assumption of LTE breaks down for hot, low gravity stars (see Fig. 2). Considerable effort has gone into developing realistic non-LTE model atmospheres for early-type stars in recent years. Ideally, temperatures are obtained using ionization equilibrium techniques, i.e. from suitable diagnostic lines of adjacent ionization stages showing a strong temperature dependence. Naturally, determinations of $T_{\text {eff }}$ from model atmospheres are critically dependent on assumptions going into those models and the accuracy with which surface gravities and elemental abundance ratios are determined. Unfortunately, due to the complexity of consistently treating metal line blanketing in extended atmospheres, i.e. considering the influence of thousands to millions of spectral lines on the atmospheric struc- 


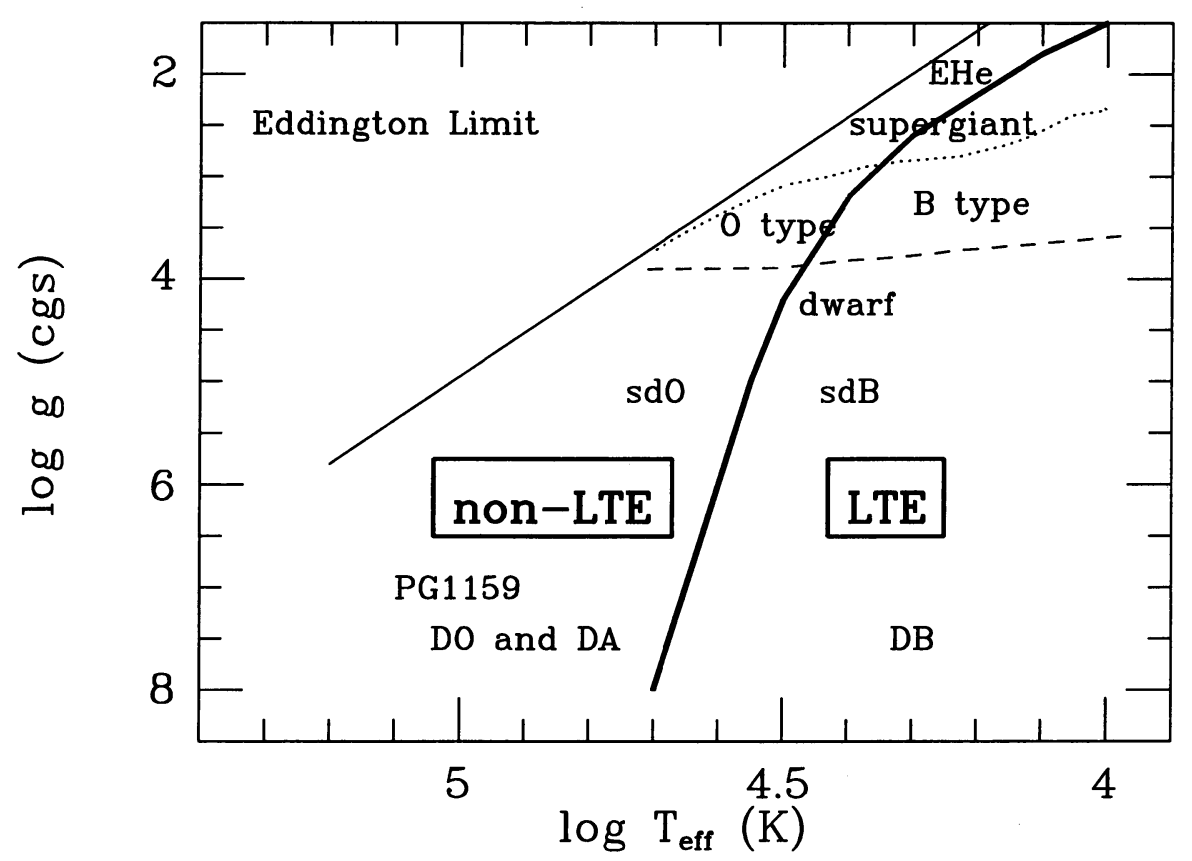

Figure 2. The position of hot stars in effective temperature and gravity discussed in this review. The Eddington limit for radiative stability is indicated (thin solid), as are the LTE and non-LTE domains (thick solid) and OB dwarf/supergiant (dashed/dotted) scales

ture (via backwarming) and emergent spectrum (via line blocking), various statistical simplifications are employed (Hillier, these proc.)

\subsection{OB DWARFS AND SUPERGIANTS}

The majority of detailed analyses of OB stars have been subject to the assumption of hydrostatic equilibrium in a plane-parallel atmosphere-(e.g. Herrero et al. 1992; Puls et al. 1996). However, should stellar winds be strong, absorption lines will be contaminated by wind effects (Schaerer \& Schmutz 1994; Crowther \& Bohannan 1997). Optical He I-II lines generally provide temperature diagnostics for $\mathrm{O}$ stars (e.g. Herrero et al. 1992) while Si II-IV lines are used for B stars (e.g. Becker \& Butler 1990), though Kudritzki (1992) discussed the complicating role of velocity fields for investigating supergiants. Kilian et al. (1991) employed both He and Si diagnostics for early B dwarfs and giants which agreed relatively well, though with differences of up to $1800 \mathrm{~K}$. Kilian et al. (1991) and Grigsby et al. (1992) used line blanketed atmospheres to obtain effective temperatures systematically higher $(2000 \mathrm{~K})$ than those from $c_{0}-T_{\text {eff }}$ photometric calibrations (e.g.

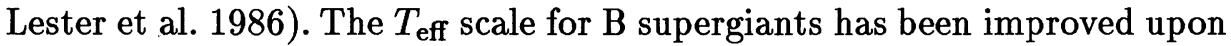


recently by McErlean et al. (1997) who used Si III-IV diagnostics for earlytypes, with non-LTE corrections to results from the Lester et al. (1986) calibration applied to mid/late B supergiants.

Recent ionization equilibrium techniques for $\mathrm{O}$ stars represent considerable advances relative to earlier (insensitive) continuum methods. For example, Bohannan et al. (1986) obtained $T_{\text {eff }}=42 \mathrm{kK}$ from a non-LTE wind blanketed analysis of $\zeta$ Pup (O4 I(n)f), with $T_{\text {eff }}=32-50 \mathrm{kK}$ obtained from continuum techniques. However, widely varying results have also been found in recent non-LTE spectroscopic analyses of late O/early B dwarfs. For instance, for HD 214680 (10Lac, O9 V) Grigsby et al. (1992) derived $T_{\text {eff }}=30.0 \mathrm{kK}$ using a line-blanketed model atmosphere, in sharp contrast to $T_{\text {eff }}=37.5 \mathrm{kK}$ from the unblanketed analysis of Herrero et al. (1992). Despite these conflicting results, HD 214680 remains widely used as a standard

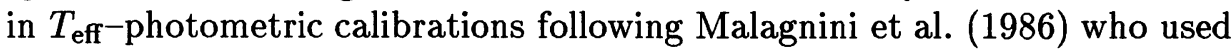
LTE Kurucz continuum fits to obtain $T_{\text {eff }}=31.75 \mathrm{kK}$. Studies based on similar non-LTE models can result in discrepant temperatures when based on optical or UV diagnostics (e.g. Melnick 42, Heap et al. 1991, Pauldrach et al. 1994). Indeed, within a single analysis different diagnostic line ratios often yield considerably different temperatures - for HD 46150 (O5 V(f)), Herrero et al. (1992) obtained $T_{\text {eff }}=42.5 \mathrm{kK}$ from He I $\lambda 4922 / \mathrm{He}$ II $\lambda 4542$ compared with $T_{\text {eff }}=47 \mathrm{kK}$ from He I $\lambda 4471 /$ He II $\lambda 4542$.

Detached OB binaries provide useful tests of spectroscopic analyses since their masses, radii, gravities can be independently and accurately determined, though temperature determinations generally rely on Strömgren photometric calibrations (Schönberner \& Harmanec 1995; Hilditch et al. 1996) which are imprecise for O and early B stars.

Various MK spectral type- $T_{\text {eff }}$ correlations have been attempted for OB stars over the past couple of decades, including Böhm-Vitense (1981), Schmidt-Kaler (1982) and Humphreys \& McElroy (1984). While these remain widely used, in ionizing flux calibrations for instance (necessary for photoionization studies of $\mathrm{H}$ II regions and starbursts), more recent scales have been obtained by Howarth \& Prinja (1989), Theodossiou \& Danezis (1991) and Vacca et al. (1996) using more recent observational and theoretical results. Due to the plethora of new results in this field we present a revised $T_{\text {eff }}$ scale of OB dwarfs and supergiants in Fig. 3, based on results from various direct, continuum and ionization equilibrium techniques. Previous standard scales are also included - note that we find considerable differences relative to the recent Vacca et al. (1996) calibration for O dwarfs since we incorporate results from blanketed studies (e.g. Voels et al. 1989; Grigsby et al. 1992) and do not impose a linear $T_{\text {eff }}$ scale. 

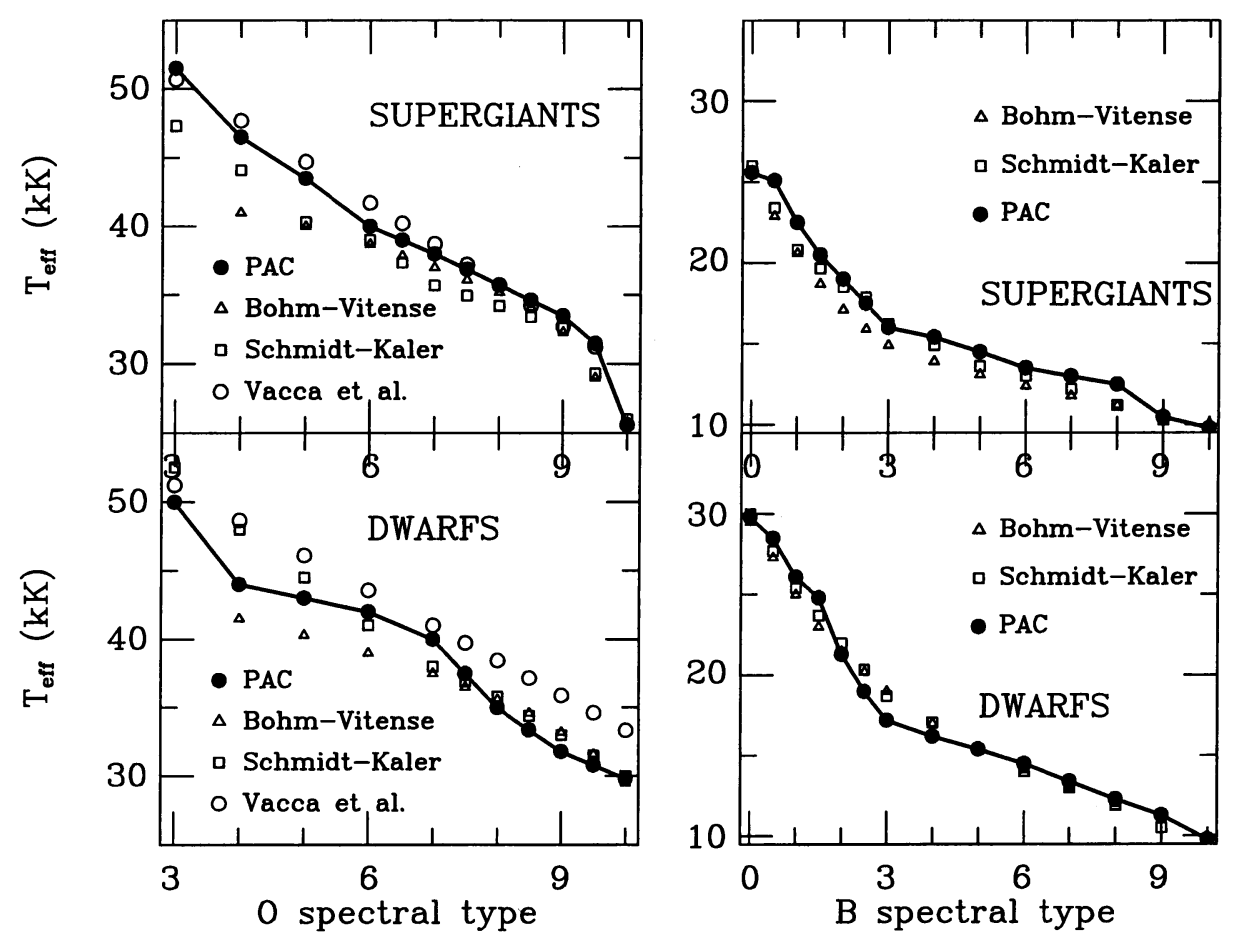

Figure 3. Comparison of our revised temperature scale for OB stars (PAC, thick solid line), available in tabular form on request, with the standard calibrations of Böhm-Vitense (1981) and Schmidt-Kaler (1982), plus the recent linear O star scale of Vacca et al. (1996)

\subsection{OB SUBDWARFS}

Recent theoretical progress in modelling $\mathrm{H}$-deficient hot subdwarfs has had a greater influence on temperature determinations than normal OB stars since the continuum opacity, dominated by hydrogen in normal stars, is severely reduced in helium stars. Therefore the line opacity is much more effective in blocking and redistributing radiation, so the treatment of lineblanketing is critical (e.g. Haas et al. 1996). In the case of He-rich sdO stars, Dreizler (1993) has used ionization equilibrium techniques with lineblanketed non-LTE models, revealing $44.5 \mathrm{kK}$ for LS IV $+10^{\circ} 9$, substantially lower than $65 \mathrm{kK}$ obtained from UV continuum methods (Schönberner \& Drilling 1984). Temperatures of sdO stars using blanketed models (Dreizler et al. 1990) are systematically $5-10 \mathrm{kK}$ lower than unblanketed results (Thejll et al. 1994).

Temperature determinations in He-poor sdB stars use Balmer line profile fits to blanketed LTE model atmospheres (e.g. Saffer et al. 1994) and are in good agreement with results from suitable Strömgren photometric 
calibrations (e.g. Bergeron et al. 1984). As with sdO stars, temperatures of H-deficient extreme helium (EHe) stars obtained from line-blanketed analyses are systematically lower $(\sim 2000 \mathrm{~K})$ than unblanketed studies (e.g. Jeffery \& Heber 1992).

\subsection{WHITE DWARFS AND PG1159 STARS}

Recent temperature determinations of hot H-rich (DA) and H-poor (DO, DB) white dwarfs and PG1159 stars are based on model Balmer line profile fits, supplemented by far/extreme UV continuum fits or ideally ionization equilibrium techniques, where observations permit.

Pure $\mathrm{H}$-atmosphere models are generally used to analyse DA white dwarfs, with temperatures obtained from optical and EUV analyses showing good consistency (see Barstow et al. 1993), and show improved temperature sensitive relative to earlier UV continuum/Ly $\alpha$ fits (e.g. Holberg et al. 1986). However, there is a well known 'Balmer line problem' for some high gravity H-rich central stars of Planetary Nebulae (CSPNe), well illustrated by Napiwotzki (1995b) who obtained discrepant temperatures using different Balmer series members for the CSPNe of the Helix nebula NGC 7293, ranging from $48 \mathrm{kK}$ (using $\mathrm{H} \alpha$ ) to $107 \mathrm{kK}(\mathrm{H} \delta)$ ! While unsolved, the incorporation of metal line blanketing improves the situation, and temperatures obtained from higher Balmer lines are in better agreement with alternative spectroscopic ( $\geq 70 \mathrm{kK}$ since He I absent) and photoionization $(120 \mathrm{kK}$ from Clegg \& Walsh 1989) results.

Napiwotzki (1995a) discussed the importance of non-LTE effects even for cool DO white dwarfs, despite their high surface gravities, while Dreizler \& Werner (1996) have compared results based on blanketed non-LTE models with previous LTE analyses finding the greatest difference between 60-70 kK. Werner et al. (1991) found that the non-LTE analyses are critical for the higher temperature PG1159 stars. In the absence of superior spectroscopic diagnostics, temperature determinations in DB white dwarfs rely on He I profile fits using LTE models (e.g. Beauchamp et al. 1995).

Stellar temperatures of CSPNe (and OB stars in $\mathrm{H}$ II regions) can also be obtained by means of their nebulae, most frequently via Zanstra analyses (Zanstra 1931; Harman \& Seaton 1966). However, results from He II are frequently inconsistent with those from H I (the 'Zanstra discrepancy') due to the use of inappropriate (often blackbody) flux distributions (Gabler et al. 1991), or an optically thin PNe. Use of realistic O-type model fluxes by Sellmaier et al. (1996) provides a possible solution to the [Ne III] problem in H II-regions, though model atmospheres are not yet fully capable of explaining both the stellar and nebular spectrum simultaneously. 


\subsection{WOLF-RAYET STARS}

Obtaining a reliable temperature scale for Wolf-Rayet (WR) stars, the chemically evolved descendents of OB stars or evolved H-deficient CSPNe, is a formidable challenge. Continuum techniques were used to estimate $T_{\text {eff }}$ in WR stars (e.g. Underhill 1983) though were shown to be unrealistic by Garmany et al. (1984). Due to extreme mass-loss, their winds are so optically thick that their photospheres are invisible, with the usual plane-parallel, LTE approximations completely invalid, with radii highly wavelength-dependent (Cherepachchuk et al. 1984). As for O stars, much progress has been made in recent years using extended non-LTE model atmospheres (e.g. Hillier 1987; Schmutz et al. 1989). Unfortunately, an added complication of their dense winds is that the usual radius at $\tau_{\text {Ross }}=2 / 3$ becomes a poor indicator of excitation in WR stars, with $\tau_{\text {Ross }}=20$ usually defining the stellar temperature, though this is difficult to relate with interior evolutionary models (Hamann 1994). Unfortunately, stellar temperatures of WR stars are also critically dependent on assumed velocity distributions (Hillier 1991), so that observational determinations of WR velocity distributions are keenly sought.

Initially, ionization balance studies restricted to lines of helium from model grids where used to obtain temperatures (e.g. Schmutz et al. 1989). These have subsequently been extended to tailored models including nitrogen in WN stars (e.g. Crowther et al. 1995a) and carbon in WC stars (e.g. Koesterke \& Hamann 1995), with heavy element line blanketed models now starting to appear. In general, results from pure helium analyses tend to underestimate stellar temperatures, with the discrepancy greater at earlier spectral type. For HD 151932 (WN7), Underhill (1983) obtained a temperature of $25 \mathrm{kK}$ from the integrated flux method, increasing to $32 \mathrm{kK}$ from non-LTE model grids by Schmutz et al. (1989) and $34 \mathrm{kK}$ from line profile fits by Crowther et al. (1995a). However, results obtained for different species in non-blanketed models generally lead to discrepant temperatures: For HD 211564 (WN3), Crowther et al. (1995b) obtained $T_{\text {eff }}=49 \mathrm{kK}$ $(69 \mathrm{kK})$ from helium (nitrogen) diagnostics! Observations of WR nebulae provide excellent tests of current theoretical flux distributions below the Lyman limit (see Esteban et al. 1993).

To summarise, huge advances have been made over the past decade towards a reliable temperature scale of hot stars. However, significant uncertainties remain, especially for $\mathrm{O}$ and WR stars, which may be resolved using nebulae as tests of theoretical energy distributions. Since real stars rotate and pulsate it is unclear what scale of temperature fluctuations are occurring across their stellar disks. 


\section{References}

Abbott D.C., Hummer D.G., 1985, ApJ 294, 286

Barstow M.A., Fleming T.A., Diamond C.J., et al. 1993, MNRAS 264, 16

Beauchamp A., Wesemael F., Bergeron P. et al. 1995, in: European Workshop on White Dwarfs, Koester D., Werner K. (eds.), Lecture Notes in Physics 433, Springer, p.108

Becker S.R., Butler K., 1990, A\&A 235, 326

Bergeron P., Fontaine G., Lacombe P. et al., 1984, AJ 89, 374

Blackwell D.E., Shallis M.J., 1977, MNRAS 180, 177

Bohannan B., Abbott D.C., Voels S.A., Hummer D.G., 1986, ApJ 308. 728

Böhm-Vitense E., 1981, ARA\&A 19, 295

Cherepachchuk A.M., Eaton J.A., Khaliullin Kh.F., 1984, ApJ 281, 774

Clegg R.E.S., Walsh J.R., 1989, in: Planetary Nebulae, Torres-Peimbert S. (ed.), Proc IAU Symp. 131, Kluwer, p.223

Code A.D., Davis J., Bless R.C., Hanbury-Brown R., 1976, ApJ 203, 417

Crowther P.A., Bohannan B., 1997, A\&A 317, 532

Crowther P.A., Smith L.J., Hillier D.J., 1995a, A\&A 293, 403

Crowther P.A., Smith L.J., Hillier D.J., 1995b, A\&A 302, 457

Dreizler S., 1993, A\&A 273, 212

Dreizler S., Werner K., 1996, A\&A 314, 217

Dreizler S., Heber U., Werner K., et al., 1990, A\&A 235, 234

Drilling J.S., Schönberner D., Heber U., Lynas-Gray A.E., 1984, ApJ 278, 224

Esteban C., Smith L.J., Vílchez J.M., Clegg R.E.S., 1993, A\&A 272, 299

Gabler R., Kudritzki R.-P., Méndez R.H., 1991, A\&A 245, 587

Garmany C.D., Massey P., Conti P.S., 1984, ApJ 278, 233

Grigsby J.A., Morrison N.D., Anderson L.S., 1992, ApJS 78, 205

Haas S., Dreizler S., Heber U., et al. 1996, A\&A 311, 669

Hamann W.-R., 1994, Space Sci. Rev. 66, 237

Harman R.J., Seaton M.J., 1966, MNRAS 132, 15

Heap S.R., Altner B., Ebbets D., et al. 1991, ApJ 377, L29

Herrero A., Kudritzki R.-P., Vilchez, J.M. et al. 1992, A\&A 261, 209

Hilditch R.W., Harries T.J., Bell S.A., 1996, A\&A 314, 165

Hillier D.J., 1987, ApJS 63, 947

Hillier D.J., 1991, in: Wolf-Rayet stars and Interrelations with Other Massive Stars in Galaxies, Proc. IAU Symp. 143, van der Hucht K.A., Hidayat B. (eds.), Kluwer, p.59

Holberg J.B., Vasile J., Wesemael, F., 1986, ApJ 306, 629

Howarth I.D., Prinja R.K., 1989, ApJS 69, 527

Hummer D.G., Abbott D.C., Voels D.A., Bohannan B., 1988, ApJ 328, 704

Humphreys R.M., McElroy D.B., 1984, ApJ 284, 565

Jeffery C.S., Heber U., 1992, A\&A 260, 133

Kilian J., Becker S.R., Gehren T., Nissen P.E., 1991, A\&A 244, 419

Koesterke L., Hamann W.-R., 1995 A\&A 299, 503

Kudritzki R.-P., 1992, A\&A 266, 395

Kudritzki R.-P., Gabler A., Gabler R., et al. 1989, in: Physics of Luminous Blue Variables, Davidson K., et al. (eds.), Kluwer, p.67

Kurucz R.L., 1979 ApJS 40, 1

Kurucz R.L., 1991, in: Precision Photometry: Astrophysics of the Galaxy, Philip A.G.D., et al. (eds.), L. Davis Press, p.27

Lester J.B., Gray R.O., Kurucz R.L., 1986, ApJS 61, 509

Malagnini M.L., Morossi C., Rossi L., Kurucz R.L., 1986, A\&A 162, 140

McErlean N., et al. 1997, in prep.

Moehler S., Heber U., 1990, De Boer K.S., A\&A 239, 265

Napiwotzki R., 1995a, in: European Workshop on White Dwarfs, Koester D., Werner K. (eds.), Lecture Notes in Physics 433, Springer, p.132

Napiwotzki R., 1995b, in: European Workshop on White Dwarfs, Koester D., Werner K. 
(eds.), Lecture Notes in Physics 433, Springer, p.176

Napiwotzki R., Schönberner D., Wenske V., 1993, A\&A 268, 653

Pauldrach A.W.A., Kudritzki R.-P., Puls J., et al. 1994 A\&A 283, 525

Puls J., Kudritzki R.-P., Herrero A. et al. 1996, A\&A 305, 171

Saffer R.A., Bergeron P., Koester D., Liebert J., 1994, ApJ 432, 351

Schaerer D., Schmutz W., 1994, A\&A 288, 231

Schmidt-Kaler Th., 1982, in: Landolt-Börnstein, Group VI, Vol 2b, Schaifers K., Voigt H.H. (eds.), Springer-Verlag

Schmutz W., Hamann W.-R., Wessolowski U., 1989, A\&A 210, 236

Schönberner D., Drilling J.S., 1984, ApJ 278, 702

Schönberner D., Harmanec P., 1995, A\&A 294, 509

Sellmaier F.H., Yamamoto T., Pauldrach A.W.A., Rubin R.H., 1996, A\&A 305, L37

Smalley B., Dworetsky M.M., 1995, A\&A 293. 446

Thejll P., Vennes S., Shipman H.L., 1991, ApJ 370, 355

Thejll P., Bauer F., Saffer R., et al. 1994, ApJ 433, 819

Theodossiou E., Danezis E., 1991, Ap\&SS 183, 91

Underhill A.B., 1983, ApJ 266, 718

Vacca W.D., Garmany C.D., Shull J.M., 1996, ApJ 460, 914

Venn K.A., 1995, ApJS 99, 659

Voels S.A., Bohannan B., Abbott D.C., Hummer D.G., 1989, ApJ 340, 107

Werner K., Heber U., Hunger K., 1991, A\&A 244, 437

Zanstra H., 1931, Publ. Don. Astrophys. Obs 4, 209

\section{DISCUSSION}

ANDRE MAEDER: Can you comment on the uncertainty in the $T_{\text {eff }}$ calibration of 0-type stars, in view of the fact that many of them show sizeable $\mathrm{He}$ and $\mathrm{N}$ enrichment?

PAUL CROWTHER: While helium enrichment in 0 stars certainly plays a role in affecting their temperature scale, uncertainties are dominated by the limited treatment of wind/line blanketing to date. More concerning is the current

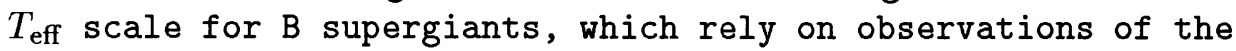
Balmer Jump (via Strömgren photometric calibrations), which is very sensitive to He-enrichment. 